\title{
Characterization of Listeria monocytogenes enhanced cold-tolerance variants isolated during prolonged cold storage
}

Hingston, Patricia A.; Hansen, Lisbeth Truelstrup; Pombert, Jean-Francois; Wang, Siyun

Published in:

International Journal of Food Microbiology

Link to article, DOI:

10.1016/j.jifoodmicro.2019.108262

Publication date:

2019

Document Version

Peer reviewed version

Link back to DTU Orbit

Citation (APA):

Hingston, P. A., Hansen, L. T., Pombert, J-F., \& Wang, S. (2019). Characterization of Listeria monocytogenes enhanced cold-tolerance variants isolated during prolonged cold storage. International Journal of Food Microbiology, 306, [108262]. https://doi.org/10.1016/j.jifoodmicro.2019.108262

\section{General rights}

Copyright and moral rights for the publications made accessible in the public portal are retained by the authors and/or other copyright owners and it is a condition of accessing publications that users recognise and abide by the legal requirements associated with these rights.

- Users may download and print one copy of any publication from the public portal for the purpose of private study or research.

- You may not further distribute the material or use it for any profit-making activity or commercial gain

- You may freely distribute the URL identifying the publication in the public portal 
1 Characterization of Listeria monocytogenes enhanced cold-tolerance variants isolated

2 during prolonged cold storage

3

4

5 Patricia A. Hingston, ${ }^{\text {a }}$ Lisbeth Truelstrup Hansen, ${ }^{\mathrm{b}}$ Jean-François Pombert, ${ }^{\mathrm{c}}$ Siyun Wang ${ }^{\mathrm{a}}$

6

7

$8{ }^{\mathrm{a}}$ Department of Food Science, University of British Columbia, Vancouver, British Columbia,

9 Canada

$10 \quad{ }^{b}$ National Food Institute, Technical University of Denmark, Kongens Lyngby, Denmark

$11{ }^{\mathrm{c} D e p a r t m e n t}$ of Biology, Illinois Institute of Technology, Chicago, Illinois, USA

14 Running Head: Traits of L. monocytogenes cold tolerant variants (54 characters allowed with

15 spaces)

16

17 \#Address correspondence to Siyun Wang, siyun.wang@ubc.ca

18

19 Abbreviations

20 BHIB, brain-heart infusion broth; ECT, enhanced cold tolerance; FA, fatty acid; FPE, food

21 processing environment; LPD, lag phase duration; $\mathrm{N}_{\max }$, maximum cell densitiy; PS, peptone

22 saline; TDR, time to detectable regrowth; SCFA, straight-chain fatty acid; SNP, single

23 nucleotide polymorphism; UFA, unsaturated fatty acid; $\mu_{\max }$, maximum growth rate 


\section{Abstract}

25 In this study, we show that growth and prolonged storage of Listeria monocytogenes at $4^{\circ} \mathrm{C}$ can

26 promote the selection of variants with enhanced cold and heat tolerance. Enhanced cold-

27 tolerance (ECT) variants $(\mathrm{n}=12)$ were successfully isolated from a strain with impaired cold

28 growth abilities following 84 days of storage at $4^{\circ} \mathrm{C}$ in brain heart infusion broth (BHIB). Whole

29 genome sequencing, membrane fatty acid analysis, and stress tolerance profiling were performed

30 on the parent strain and two ECT variants: one displaying regular-sized colonies and the other

31 displaying small colonies when grown at $37^{\circ} \mathrm{C}$ on $\mathrm{BHI}$ agar. Under cold stress conditions, the

32 parent strain exhibited an impaired ability to produce branched-chain fatty acids which are

33 known to be important for cold adaptation in L. monocytogenes. The ECT variants were able to

34 overcome this limitation, a finding which is hypothesized to be associated with the identification

35 of two independent single-nucleotide polymorphisms in genes encoding subunits of acetyl-coA

36 carboxylase, an enzyme critical for fatty acid biosynthesis. While the ECT phenotype was not

37 found to be associated with improved salt (BHIB $\left.+6 \% \mathrm{NaCl}, 25^{\circ} \mathrm{C}\right)$, acid $\left(\mathrm{BHIB} \mathrm{pH} 5,25^{\circ} \mathrm{C}\right)$ or

38 desiccation $\left(33 \% \mathrm{RH}, 20^{\circ} \mathrm{C}\right)$ tolerance, the small-colony variant exhibited significantly $(\mathrm{p}<0.05)$

39 enhanced heat tolerance at $52^{\circ} \mathrm{C}$ in buffered peptone water compared to the parent strain and the

40 other variant. The results from this study demonstrate that the continuous use of refrigeration

41 along the food-supply chain has the potential to select for $L$. monocytogenes variants with

42 enhanced cold and heat tolerance, highlighting the impact that microbial intervention strategies

43 can have on the evolution of bacterial strains and likewise, food safety.

\section{$44 \quad$ Keywords}

45 Whole-genome sequencing; cold tolerance; membrane-lipid profiling; stress-tolerance profiling; 46 single-nucleotide polymorphisms 


\section{Introduction}

The human pathogen Listeria monocytogenes represents an ongoing concern in the food

49 industry globally, where it is continuously detected in food products leading to costly recalls,

50 loss of consumer trust and considerable public health concerns. Furthermore, while $L$.

51 monocytogenes outbreaks have traditionally been associated with fresh produce and other ready-

52 to-eat products such as deli meats and cheeses, more recent outbreaks in North America have

53 implicated new food vectors including candy apples and ice cream (US CDC, 2017). The

54 occurrence of these unexpected outbreaks demonstrates that there is still a great deal to be learnt

55 regarding this foodborne pathogen and the factors facilitating its survival and/or growth in both

56 foods and food-processing environments.

57 While L. monocytogenes is mostly recognized for its ability to grow at refrigeration

58 temperatures, it is also capable of tolerating a number of other food-related stresses. Notably, it

59 has been shown to grow in the presence of up to $12 \%$ salt and at pH levels as low as 4.7 (Cole et

60 al., 1990; Walker et al., 1990). However, bacteria are known to differ in their abilities to tolerate

61 various stresses, meaning that the reported limits only represent the abilities of the specific

62 strains evaluated in these studies. Differences between strain phenotypic behavior commonly

63 stem from the presence/absence of chromosomally or plasmid located genes, or from single

64 nucleotide polymorphisms (SNPs) which can occur as errors during replication or as a result of

65 horizontal gene transfer or a selective pressure such as antibiotics. While disadvantageous

66 bacterial mutations are approximately 100,000× more common than beneficial mutations (e.g.,

67 estimated to be $\sim 10^{-4}$ mutations per genome per replication vs. $10^{-9}$ for adverse and beneficial

68 mutations, respectively, in Escherichia coli) (Boe et al., 2000; Imhof and Schlotterer, 2001),

69 beneficial mutations remain a large concern for the food industry as arising strains may possess 
70 enhanced survival capabilities that render current intervention and safety measures ineffective.

71 Given the importance of preventing L. monocytogenes from reaching unsafe levels in food, little

72 is known regarding conditions that may select for variants with enhanced abilities to tolerate

73 food-related stresses.

74 In a previous study, we screened 166 L. monocytogenes strains for their ability to tolerate

75 cold, acid, salt, and desiccation stress, and then used whole-genome sequencing to identify

76 genetic elements common among stress-tolerant and -sensitive phenotypes (Hingston et al.,

77 2017b). While some common genetic elements were identified among stress-sensitive strains, no

78 common genomic footprints were identified among stress-tolerant phenotypes, suggesting

79 homoplasy where mutations evolve independently to confer tolerance. Another important finding

80 from this study was that closely related isolates from within the same sequence type (multilocus

81 sequence typing) exhibited opposing stress tolerances, suggesting that minor genetic differences

82 can exert great impact on stress-tolerance phenotypes.

83 As listeriosis outbreaks are most commonly associated with refrigerated, ready-to-eat

84 foods, the objectives of this study were to 1) determine if prolonged cold-stress exposure can

85 promote the formation of $L$. monocytogenes variants with enhanced cold tolerance (ECT), 2)

86 uncover potential mutations associated with ECT, and 3) determine whether these mutations are

87 associated with enhanced tolerances to other food-related stresses.

\section{Materials and methods}

\subsection{Bacterial strains and culture conditions}

90 A selection of $11 \mathrm{~L}$. monocytogenes food-related strains (Table 1) previously characterized

91 for food-related stress tolerances and subjected to whole-genome sequencing (Hingston et al., 
92 2017b), were used in this study. Strains were assigned as being stress sensitive or tolerant based

93 on having a maximum growth rate at $4^{\circ} \mathrm{C}$ that was at least one standard deviation smaller or

94 larger than the median value for all strains, respectively. All remaining isolates were considered

95 to have intermediate stress tolerance. The median was selected for standardization rather than the

96 mean to avoid the influence of very stress sensitive isolates. Based on the hypothesis that faster

97 cold-growing strains evolved from slower growing wild type strains, four cold-sensitive strains

98 and six intermediate cold-tolerance strains covering a range of $L$. monocytogenes serotypes were

99 selected for this study. Additionally, a single fast cold-growing (cold tolerant) strain (Lm1)

100 whose mechanisms of cold stress survival have been thoroughly investigated (Hingston et al.,

101 2017a), was also included for comparison. Strains were stored at $-80^{\circ} \mathrm{C}$ in brain heart infusion

102 broth (BHIB, Difco, Fisher Scientific, Ottawa, Canada) $+20 \%(v / v)$ glycerol, and routinely

103 cultured at $37^{\circ} \mathrm{C}$ on BHI agar (Difco, Fisher Scientific) plates. 
Table 1. L. monocytogenes strains included in the long-term cold stress exposure study.

\begin{tabular}{|c|c|c|c|c|c|c|c|}
\hline $\begin{array}{c}\text { Strain } \\
\text { name }\end{array}$ & $\begin{array}{l}\text { Province or } \\
\text { country of } \\
\text { origin }\end{array}$ & Sample date & Source & Serotype & $\begin{array}{l}\text { Sequence } \\
\text { type }\end{array}$ & $\begin{array}{c}\text { Cold } \\
\text { tolerance } \\
\text { phenotype }\end{array}$ & $\begin{array}{c}\text { BioSample accession } \\
\text { number in GenBank } \\
\text { (BioProject } \\
\text { PRGNA329415) }\end{array}$ \\
\hline Lm1 & $\mathrm{BC}$ & Aug - Oct 2009 & FPE & $1 / 2 \mathrm{a}$ & 7 & Tolerant & SAMN05256775 \\
\hline $\operatorname{Lm} 20$ & $\mathrm{BC}$ & Aug - Oct 2009 & FPE & $1 / 2 c$ & 9 & Intermediate & SAMN05410574 \\
\hline $\operatorname{Lm} 22$ & $\mathrm{BC}$ & Aug - Oct 2009 & FPE & $3 a$ & 321 & Intermediate & SAMN05410576 \\
\hline Lm32 & $\mathrm{BC}$ & Aug - Oct 2009 & FPE & $1 / 2 a$ & 155 & Intermediate & SAMN05410578 \\
\hline Lm60 & $\mathrm{BC}$ & Aug - Oct 2009 & Food & $4 \mathrm{~b}$ & 194 & Intermediate & SAMN05410586 \\
\hline $\operatorname{Lm70}$ & $\mathrm{BC}$ & Aug - Oct 2009 & FPE & $4 \mathrm{~b}$ & 6 & Intermediate & SAMN05410588 \\
\hline Lm87 & $\mathrm{BC}$ & Aug - Oct 2009 & Food & $4 \mathrm{~b}$ & 6 & Intermediate & SAMN05410636 \\
\hline Lm96 & $\mathrm{BC}$ & Aug - Oct 2009 & FPE & $1 / 2 \mathrm{a}$ & 7 & Sensitive & SAMN05410647 \\
\hline Lm231 & $\mathrm{CH}$ & 2004 & $\begin{array}{l}\text { Asymptomatic } \\
\text { Human Carriage }\end{array}$ & $1 / 2 c$ & 9 & Sensitive & SAMN05410726 \\
\hline Lm233 & $\mathrm{CH}$ & 2002 & Food & $1 / 2 c$ & 9 & Sensitive & SAMN05410734 \\
\hline LmA5 & $\mathrm{AB}$ & Aug 1990 & Food & $4 \mathrm{~b}$ & 1 & Sensitive & SAMN05256773 \\
\hline
\end{tabular}

$105 \mathrm{BC}=$ British Columbia, Canada; $\mathrm{AB}=$ Alberta, Canada; $\mathrm{CH}=$ Switzerland

$106 \quad \mathrm{FPE}=$ food processing environment

107 Sequence types were previously determined (Hingston et al., 2017b) via multilocus sequence typing

108 

storage at $4^{\circ} \mathrm{C}$

A single colony from each strain was inoculated into BHIB and grown for $24 \mathrm{~h}$ at $20^{\circ} \mathrm{C}$.

115 The cultures were then diluted in $10 \mathrm{~mL}$ of fresh BHIB to a concentration of $10^{7} \mathrm{CFU} / \mathrm{mL}$ and 116 stored for 389 days at $4^{\circ} \mathrm{C}$. Six replicates were prepared for each strain. Cultures were routinely

117 enumerated by diluting in peptone saline [PS; 0.1\% bacteriological peptone (Oxoid, Fisher 118 Scientific), 0.85\% NaCl], plating on tryptic soy agar (TSA; BD, Fisher Scientific) + 6\% yeast 119 extract (YE; BD, Fisher Scientific) and incubating for $48 \mathrm{~h}$ at $37^{\circ} \mathrm{C}$. Four of the six tubes were 120 used to monitor the $\mathrm{pH}$ of the broth over time. The $\mathrm{pH}$ of the filter sterilized $(0.2 \mu \mathrm{m}$ pore size $)$ 121 broth was measured after 5 days (exponential phase), 10 days (stationary phase), 16 days (late122 stationary phase), and 245 days (death phase) of incubation at $4^{\circ} \mathrm{C}$.

\subsection{Screening for $L$. monocytogenes variants with enhanced cold tolerance}

124 At seven time points throughout the 389 days of cold storage, a $100 \mu \mathrm{L}$ aliquot from each

125 culture was removed and stored at $-80^{\circ} \mathrm{C}$ with the addition of $20 \%(\mathrm{v} / \mathrm{v})$ glycerol. The first

126 freeze-down took place 53 days following the start of the experiment and the remaining six

127 freeze-down dates were spaced a month to a month and a half apart with the final freeze-down 128 date taking place on day 297 (Figure 1A).

129 After the end of the experimental period, the seven frozen aliquots per strain were 130 streaked out onto BHI agar (incubated at $37^{\circ} \mathrm{C}$ for $24 \mathrm{~h}$ ) and two colonies were randomly 131 selected from each plate to conduct cold growth comparisons relative to the parent strains. To do 132 this, the colonies were grown in BHIB for $24 \mathrm{~h}$ at $20^{\circ} \mathrm{C}$ and then diluted in fresh $\mathrm{BHIB}$ to a 133 concentration of $10^{3} \mathrm{CFU} / \mathrm{mL}$ and stored at $4^{\circ} \mathrm{C}$ for up to five weeks. The cell densities of the 
134 cultures were enumerated daily for the first four days and then bi-weekly thereafter. The

135 resulting growth curves were fitted using a four parameter logistic model described by Dalgaard

136 and Koutsoumanis (Dalgaard and Koutsoumanis, 2001) and the maximum growth rates $\left(\mu_{\max }\right)$

137 and final cell densities $\left(\mathrm{N}_{\max }\right)$ were compared between the long-term cold storage isolates and the

138 parent strains. The Dalgaard and Koutsoumanis (2001) model was selected for this assay because

139 it is more accommodating of fewer sampling points compared to other microbial growth models

140 such as the Baranyi and Roberts model (Baranyi and Roberts, 1994) which is later used in this

141 study to model spectrophotometrically obtained data with frequent sampling points.

142 An additional screening method was also employed where $10 \mu \mathrm{L}$ from each frozen

143 aliquot was directly regrown in $10 \mathrm{~mL}$ of $\mathrm{BHIB}$ at $4^{\circ} \mathrm{C}$ until stationary phase was reached

144 (approx. 10-14 days) with the aim of selecting for faster cold-growing strains. Once the cultures

145 reached stationary phase at $4^{\circ} \mathrm{C}, 10 \mu \mathrm{L}$ of the culture was inoculated into $10 \mathrm{~mL}$ of fresh BHIB

146 and regrown a second time at $4^{\circ} \mathrm{C}$. This procedure was repeated a total of three times. Each of

147 the three resulting regrowth curves was modeled as previously described, and the $\mu_{\max }$ and $N_{\max }$

148 values were compared to those of the parent strains.

$149 \quad$ 2.4. Stress-tolerance profiling

150 To determine if isolated ECT variants possessed enhanced tolerances to other food-related 151 stresses, two variants displaying different colony morphologies (Table 2) were selected for 152 further stress-tolerance profiling along with the wild type strain. Salt, acid, and desiccation 153 tolerance were evaluated using previously described protocols (Hingston et al., 2017b). In short, 154 cultures were originally grown in $\mathrm{BHIB}$ at $30^{\circ} \mathrm{C}$, resuspended to $10^{7} \mathrm{CFU} / \mathrm{mL}$ in fresh $\mathrm{BHIB}+6 \%$ $155 \mathrm{NaCl}$ or BHIB adjusted to $\mathrm{pH} 5$ in 96-well plates (Costar ${ }^{\mathrm{TM}}$ clear polystyrene, Fisher Scientific) 
156 and incubated at $25^{\circ} \mathrm{C}$ in a microplate reader (Spectramax, V6.3; Molecular Devices, Sunnyvale, 157 California, USA) (set at $600 \mathrm{~nm}$ ) until all cultures reached stationary phase ( 26 h). A 158 temperature of $25^{\circ} \mathrm{C}$ was used to assess strain salt and acid tolerance under non-intracellular or 159 cold stress conditions.

160 Desiccation survival was evaluated by growing cultures in BHIB at $20^{\circ} \mathrm{C}$, diluting to $10^{7}$

$161 \mathrm{CFU} / \mathrm{mL}$ in buffered peptone water (BD, Fisher Scientific), and spotting $10 \mu \mathrm{L}\left(10^{5} \mathrm{CFU}\right)$ in lid162 less 96-well plates that were then stored for four days at $20^{\circ} \mathrm{C}$ in desiccators (SICCO, Bohlender, 163 Germany) pre-conditioned to 33\% relative humidity (RH). Following desiccation, the plates 164 were rehydrated with $\mathrm{BHIB}$, and incubated at $25^{\circ} \mathrm{C}$ in a plate reader (set at $600 \mathrm{~nm}$ ) until all 165 cultures reached stationary phase $(\sim 24 \mathrm{~h})$. A temperature of $20^{\circ} \mathrm{C}$ was used for both culturing and 166 desiccating cells to mimic a situation that may occur in a food plant, while $25^{\circ} \mathrm{C}$ was used for 167 regrowth following desiccation, so that the growth curve parameters results from the salt, acid, 168 and desiccation stress experiments could be compared. 
169 Table 2. L. monocytogenes enhanced cold-tolerance variants characterized in this study.

\begin{tabular}{lccccc}
\hline \multicolumn{1}{c}{ Strain } & Colony size* & BioProject & $\begin{array}{c}\text { BioSample accession } \\
\text { numbers }\end{array}$ & $\begin{array}{c}\text { NCBI GenBank } \\
\text { accession numbers }\end{array}$ & $\begin{array}{c}\text { NCBI Sequence Read } \\
\text { Archive accession } \\
\text { numbers }\end{array}$ \\
\hline Lm96_84d & Regular & PRJNA480160 & SAMN09629801 & GCA_003344745.1 & SRR7496279 \\
Lm96_84d_sm & Small & & SAMN09629803 & GCA_003344725.1 & SRR7496280 \\
\hline * On brain heart infusion agar incubated at 37 C. & & &
\end{tabular}

$170 *$ On brain heart infusion agar incubated at $37^{\circ} \mathrm{C}$. 
172 2015). The parent strain and two variants were grown for $24 \mathrm{~h}$ in $\mathrm{BHIB}$ at $20^{\circ} \mathrm{C}$, diluted to $10^{7}$

$173 \mathrm{CFU} / \mathrm{mL}$ in PS, and then $50 \mu \mathrm{L}$ aliquots were placed in a thermocycler set at $52^{\circ} \mathrm{C}$. Survivors

174 were enumerated on BHI agar after 0, 15, 30, 45, and 60 min.

175 Three biological replicates of each strain were conducted for the salt, acid and heat

176 tolerance assays whereas six biological replicates were used in the desiccation tolerance assay as

177 the experiment produced higher levels of variance. The resulting growth curves from the salt,

178 acid, and desiccation tolerance assays were fitted to the Baranyi and Roberts model (Baranyi and

179 Roberts, 1994) using DMfit (v3.5) available on the ComBase browser

180 (http://browser.combase.cc/DMFit.aspx). Model parameters were statistically compared among

181 strains using one-way ANOVAs with Tukey post-hoc tests provided by SPSS statistical software

182 (https://www.ibm.com/analytics/data-science/predictive-analytics/spss-statistical-software).

183 Strain heat tolerances were also analyzed using one-way ANOVAs with Tukey post-hoc tests to 184 compare the log reductions in survival at each of the sampling-times. Results with a p-value $185<0.05$ were considered significant in all cases.

\subsection{Cell morphology and colony enumeration}

A single colony from the parent strain and the two ECT variants was suspended in PS

188 on slides, heat fixed, and stained with crystal violet. The slides were visualized under 1000× 189 magnification using an AxioCam camera (Zeiss, Germany) attached to an Axioskop 2 mot plus 190 phase-contrast microscope (Zeiss), and the lengths of 15 cells (per strain) were measured using 191 AxioVision software (Zeiss). 
193 cultures were appropriately diluted, spread plated on TSA-YE, and incubated for $24 \mathrm{~h}$ at $37^{\circ} \mathrm{C}$.

194 Following incubation, three colonies from each plate were removed from the agar surface using

195 trimmed $200 \mu \mathrm{L}$ pipette tips and transferred to a microcentrifuge tube containing $1 \mathrm{~mL}$ of PS.

196 The tubes were thoroughly vortexed and then enumerated as previously described.

\subsection{Membrane lipid profiling}

Membrane-lipid profiling was performed on exponential-phase cultures of two ECT variants and the parent strain grown at $20^{\circ} \mathrm{C}$ and $4{ }^{\circ} \mathrm{C}$ in BHIB. Cultures were pelleted (10 - $\left.45 \mathrm{mg}\right)$,

200 rinsed twice with $1 \mathrm{~mL}$ of PS, and stored at $-80^{\circ} \mathrm{C}$. Additional frozen pellets were also prepared

201 from exponential-phase cultures of three non-ECT variant strains from the long-term cold 202 storage experiment (Lm1, Lm20, Lm231). This was performed to investigate whether long-term 203 cold stress survival is associated with a specific membrane lipid profile. To complete this 204 objective, the long-term cold-storage cultures of the three aforementioned strains were pelleted 205 after 354 days of cold storage. All frozen pellets were later sent to MIDI labs (Microbial ID, Inc.,

206 Newark, Denver, USA) where cell lipids were extracted, and fatty acids were subsequently 207 methylated for gas chromatography analysis. The resulting fatty acid methyl ester (FAME) 208 profiles were then analyzed using Sherlock ${ }^{\circledR}$ pattern recognition software.

\subsection{Whole genome sequencing and SNP analysis}

210 Genomic DNA samples from strain Lm96 and two cold tolerant variants isolated after 84

211 days of cold storage were isolated using the PureLink Mini Kit from Life Technologies (Fisher 212 Scientific). dsDNA quantification was performed using a PicoGreen assay kit from Invitrogen 213 (Fisher Scientific) and DNA quality was assessed using the NanoDrop 2000 (Fisher Scientific). 
214 Genomic DNA samples of sufficient quality and quantity were sequenced by Genome Quebec

215 (Montréal, QC, Canada) using shotgun library preparation and paired-end, 125 bp sequencing on

216 the Illumina Hi-Seq 2500 platform. Over two million reads were generated for each sample, with

217 a minimum sequence depth of 182x. de novo genome assembly was performed using SPAdes

218 version 3.10.1 (Bankevich et al., 2012). Assemblies were subsequently annotated via the NCBI

219 prokaryotic genome annotation pipeline (Tatusova et al., 2016).

220 To identify single nucleotide polymorphisms and insertions/deletions between strain

221 Lm96 and cold tolerant variants, Illumina sequencing reads were mapped against the reference

222 Lm96 genome assembly using the SSRG pipeline (https://github.com/PombertLab/SNPs) as

223 follows. Reads were mapped against the Lm96 reference genome using Bowtie 2 version 2.3.1

224 (Langmead and Salzberg, 2012) in paired-ends mode with as maximum insert distance of 1,000

225 nt. Variants were called from the aligned reads using VarScan2 version 4.1 (Koboldt et al., 2012)

226 with the number of minimum supporting reads (-mr) set to 15 and the minimum variant allele

227 frequency -mvf set to 0.7 to discard artefacts cause by reads mapping to paralogous genes. Reads

228 from Lm96 were also mapped against the Lm96 assembly to further detect false positives.

229 Synonymous and non-synonymous mutations against the Lm96 reference genome were inferred

230 from the variants in the VarScan VCF files with synonymy.pl from the SSRG pipeline.

\section{3. Results and discussion}

$232 \quad$ 3.1. Prolonged storage of $L$. monocytogenes cultures at $4^{\circ} \mathrm{C}$ was associated with the appearance of pinpoint colonies and a decrease in $\mathrm{pH}$ and survival

235 decreased from $9 \log$ CFU/mL to $5.2-7.3 \log$ CFU/mL (Fig 1A) for all but one of the 11 strains. 
This equates to viable cell reductions of $1.8-3.7 \log \mathrm{CFU} / \mathrm{mL}$ or $0.02-1.53 \%$ survival. For the

237 one remaining strain, regrowth was observed after 283 days that resulted in $8.8 \log$ CFU/mL

238 viable cells after 389 days. Following 10 days of cold storage, the $\mathrm{pH}$ of all strain cultures

239 decreased from $7.21 \pm 0.01$ to $5.82 \pm 0.03$, subjecting the bacterial cells to acid stress in addition to

240 the existing cold stress. After 263 days of cold storage the $\mathrm{pH}$ of the cultures further decreased to

$2415.67 \pm 0.03$. This slight but continuous reduction in $\mathrm{pH}$ demonstrates that despite an overall

242 decrease in survival, some cells remained metabolically active throughout the prolonged storage

243 at $4^{\circ} \mathrm{C}$.
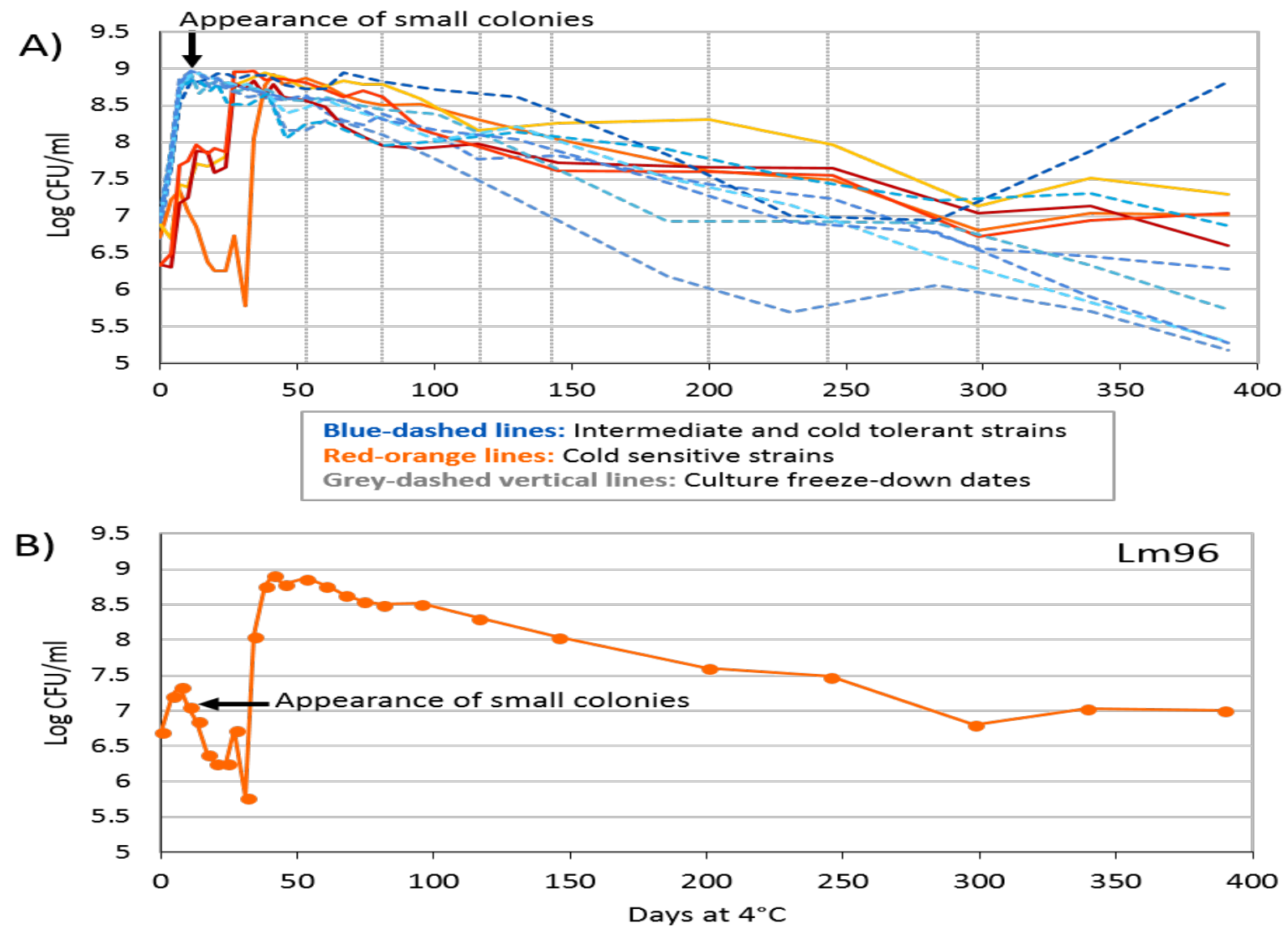

Figure 1. Growth and survival of L. monocytogenes strain cultures throughout 389 days of storage at $4^{\circ} \mathrm{C}$ in brain heart infusion broth $(n=1)$. A) Cold sensitive and intermediate cold tolerant strains, B) Lm96, the cold sensitive parent strain of enhanced cold-tolerance variants. 
250 tolerant strains. After 10 days at $4^{\circ} \mathrm{C}$, all intermediate cold-tolerant strains were able to reach a

251 maximum cell density of $9 \log$ CFU/mL from a starting concentration of 7 log CFU/mL (Fig

252 1A). However, this trend was not observed for the cold-sensitive strains (Lm231, Lm233,

253 LmA5) who after 10 days at $4^{\circ} \mathrm{C}$, reached a cell density of only $\sim 7.7 \log \mathrm{CFU} / \mathrm{mL}$ and

254 maintained this level for approx. 10 days. After this time, cell numbers began to increase again,

255 finally reaching a maximum level of $9 \log$ CFU/mL like the intermediate cold-tolerant strains

256 (Fig 1A). The fourth cold-sensitive strain (Lm96) followed a similar trend; however, instead of 257 displaying regrowth after the first initial plateau at $7.7 \log$ CFU/mL, viable cell counts dropped 258 to $5.8 \log \mathrm{CFU} / \mathrm{mL}$ by day 31 (Fig 1A and B). By day 41 however, the culture unexpectedly 259 produced viable cell counts of $8.92 \log$ CFU/mL (Fig 1B). Possible explanations for this event 260 will be discussed in the following section.

261 Traditionally, the bacterial life cycle is known to consist of three growth phases: lag 262 phase, exponential phase, and stationary phase. However, when batch cultures are incubated for 263 longer periods of time two additional phases are also observed: death phase and long-term 264 stationary phase (Finkel, 2006). It is not yet completely understood why cell death occurs 265 following stationary phase but it is generally accepted that after a given period of time, the 266 nutrients in a particular environment become depleted and cells can no longer carry out repair 267 and maintenance functions and therefore begin to die. Dead cells can then be deconstructed and 268 used as nutrients for other cells, allowing a subpopulation to survive in what has been described 269 as extended or long-term stationary phase (Finkel, 2006). Bruno and Freitag (2011) showed in a 27012 day long experiment, that following $1-2$ days of stationary phase at $37^{\circ} \mathrm{C}$ in $\mathrm{BHIB}, L$. 271 monocytogenes cultures exhibited a $24 \mathrm{~h}$ death phase followed by a long-term stationary phase 
272 with populations of $10^{7} \mathrm{CFU} / \mathrm{mL}$. E. coli has similarly been shown to maintain a cell density of $273 \sim 10^{6} \mathrm{CFU} / \mathrm{mL}$ for more than five years at $37^{\circ} \mathrm{C}$ in $\mathrm{LB}$ broth without the addition of nutrients 274 (Finkel and Kolter, 1999).

275 In the present study, decreases in cell viability occurred approximately 4 days following 276 the onset of stationary phase at $4^{\circ} \mathrm{C}$, with more notable reductions $(>0.5 \log \mathrm{CFU} / \mathrm{mL})$ observed 277 50-60 days thereafter. The onset of long-term stationary phase is more difficult to determine as 278 generally speaking, the number of viable cells in all cultures continued to decrease throughout 279 the 389 days of $4^{\circ} \mathrm{C}$ storage. One exception was Lm20, which exhibited an increase in viability 280 following $\sim 283$ days at $4^{\circ} \mathrm{C}$ (Fig $\left.1 \mathrm{~A}\right)$.

281 Long-term stationary phase studies commonly report the presence of different colony 282 morphologies ranging from pinpoint-sized colonies to fried-egg colonies with ruffled edges and 283 darker centers (Finkel, 2006; Zinser and Kolter, 2004). Pinpoint or small-colony variants are 284 frequently reported in studies where bacteria are enumerated following exposure to a stress 285 (Kahl, 2014; Ochiai et al., n.d.) and are a result of longer lag phase durations as cells recover 286 (Cooper et al., 1968). Accordingly, stress-induced small-colonies are nonstable and revert back 287 to their regular size upon sub culturing (Leimer et al., 2016). In the present study, a mix of 288 regular-sized and small colonies at an approximately 1:1 ratio were visible on the agar plates of 289 all strain cultures after around 18 days at $4^{\circ} \mathrm{C}$, demonstrating that the cells were experiencing 290 stress. However, it is interesting to note that after approximately 265 days of $4^{\circ} \mathrm{C}$ storage, many 291 of the cultures (Lm1, Lm96, Lm225, Lm231, Lm233, Lm296, Lm60) began to revert back to 292 producing all regular-sized colonies. This switch may indicate either changes in the overall 293 health of the cells as dead cells provide alternative energy sources for them to consume, or the 294 presence of new variants that have overtaken the populations. In either case, this observation 
may be associated with the re-growth observed for Lm20 following 283 days of $4^{\circ} \mathrm{C}$ storage. As

296 no ECT variants were successfully isolated from this strain culture (up to 293 days at $4^{\circ} \mathrm{C}$

297 storage), the exact reason for the increase in cell viability after 283 days remains unknown.

\subsection{ECT variants were successfully isolated from the culture of a cold sensitive $L$.} monocytogenes strain

Twelve L. monocytogenes variants with ECT were successfully isolated from the $4^{\circ} \mathrm{C}$

301 prolonged storage culture of Lm96, a cold-sensitive strain. Since two colonies were screened

302 from frozen-aliquots collected at seven different time points, these 12 ECT variants represent $30388 \%(12 / 14)$ of the total isolates tested from this culture. The two isolates screened from the 304 aliquot collected after 54 days of $4^{\circ} \mathrm{C}$ storage did not exhibit an ECT phenotype. The $100 \%$ 305 success rate of isolating ECT variants after 84 days implies that all or a very high proportion of 306 the cells in the Lm96 culture exhibited this phenotype.

307 Apart from screening the cold growth abilities of individual colonies, a second screening 308 technique was also employed that involved repeatedly transferring and re-growing the 309 community of cells from each frozen aliquot, at $4^{\circ} \mathrm{C}$ in BHIB. This approach aimed to enrich for 310 the presence of ECT variants in cultures where individual colony screening was not successful. 311 However, even after three consecutive transfers, all growth profiles from the "enriched" 312 community cultures matched those of the parent strains (data not shown).

313 All ECT variants had near-identical growth profiles at $4^{\circ} \mathrm{C}$, obtaining maximum cell 314 densities of $8.96 \pm 0.02 \mathrm{CFU} / \mathrm{mL}$ compared to $7.21 \pm 0.10 \log \mathrm{CFU} / \mathrm{mL}$ observed for the parent 315 strain (Fig 2). Similarly, the parent strain had a maximum growth rate $\left(\mu_{\max }\right)$ of $0.58 \pm 0.03 \log$ $316 \mathrm{CFU} / \mathrm{mL} / \mathrm{h}$ while the ECT variants had a significantly $(\mathrm{p}<0.05)$ higher $\mu_{\max }$ of $0.94 \pm 0.02$ (Fig 2 ). 


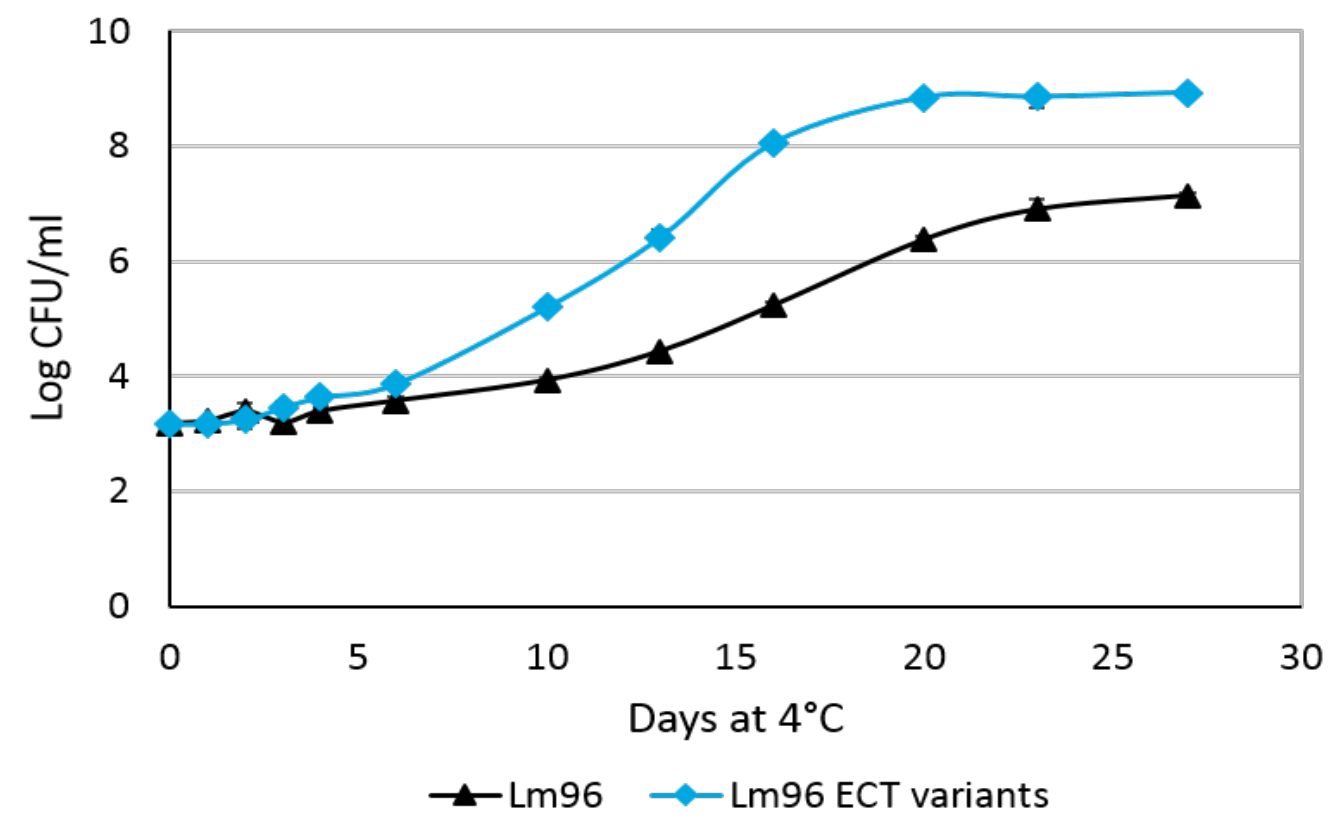

318 Figure 2. Growth comparisons of Lm96, enhanced cold-tolerance (ECT) variant Lm96_84d_sm, 319 and an intermediate (Lm20) and cold tolerant (Lm1) strain at $4^{\circ} \mathrm{C}$ in brain heart infusion broth. 320 Since a batch effect was observed across replicates, one replicate of each strain is shown above. 321 All ECT variants $(\mathrm{n}=12)$ exhibited similar growth profiles and are represented here by 322 Lm96_84d_sm.

For comparison purposes, the growth curves of a cold tolerant (Lm1) and intermediate 325 (Lm20) strain from our previous study (Hingston et al., 2017b) were also included in Fig 2. 326 Compared to these strains, the ECT variants still had longer lag phase durations ( $>0.05)$, 327 however, the maximum growth rates and cell densities were the same ( $>>0.05)$. The ability of the 328 ECT variants to reach a maximum cell density 2 log higher than the wild type strain suggests the 329 presence of such variants in food could pose a higher threat to consumer food safety relative to 330 the presence of the wild type strain. Moreover, given that after 18 days the variants were able to 331 reach the same density of cells as the cold tolerant strain, Lm1, the ECT variants could pose the 332 same level of threat to consumers as cold tolerant strains depending on the shelf life of a product 333 and when it is consumed. 

with the onset coinciding with the first drop in cell viability (Fig 1B). Unique to Lm96 was that the small colonies maintained their morphology throughout multiple transfers at $37^{\circ} \mathrm{C}$ while the small colonies observed from all other prolonged $4^{\circ} \mathrm{C}$ storage cultures reverted back to their regular-size. Six of the ECT variants exhibited this permanent small-colony morphology while

339 the other six displayed regular-sized colonies (Fig 3AB).

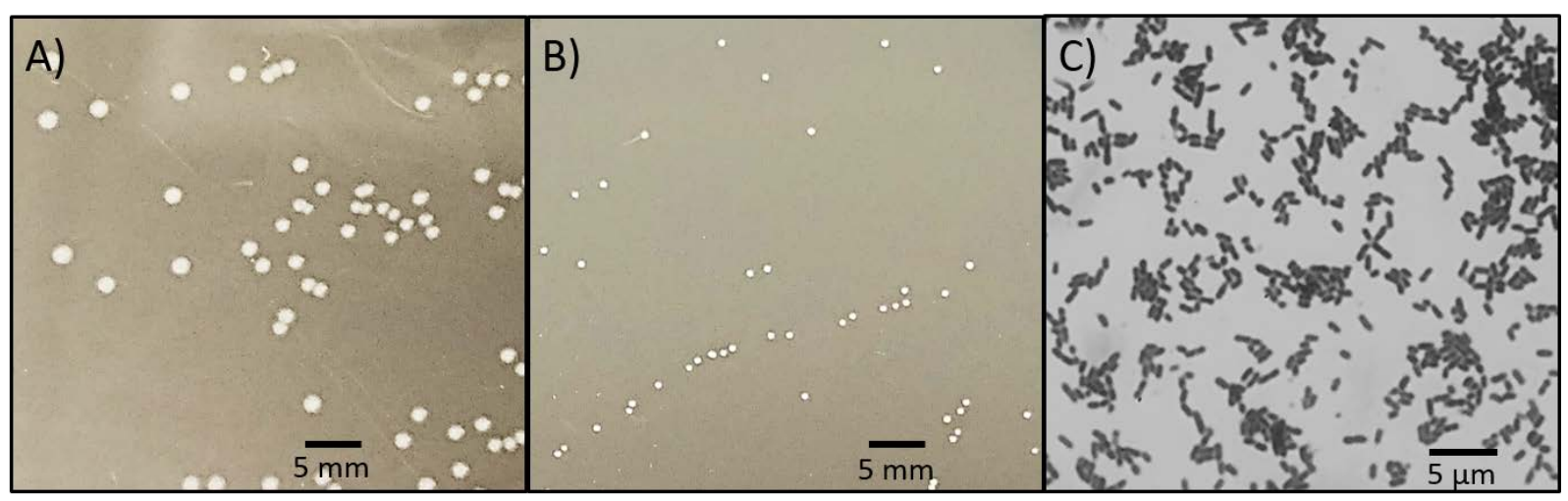

342 Figure 3. Colony morphology representations of A) Lm96 and Lm96_84d, and B) 343 Lm96_84d_sm. C) Cellular morphology representation of Lm96, Lm96_84d, and 344 Lm96_84d_sm. Colony morphologies were captured on brain heart infusion agar plates 345 following 24 hours of incubation at $37^{\circ} \mathrm{C}$.

To determine if the small colonies were a result of smaller cells or a decreased ability to

347 grow on the agar plates at $37^{\circ} \mathrm{C}$, both cell sizes and the number of cells in each colony type were 348 compared between the parent strain and the ECT variants. No differences ( $>0.05)$ in cell length

349 were detected $(0.89 \pm 0.14 \mu \mathrm{m})$, however, the small-colony variants did contain significantly $350 \quad(p<0.0005)$ fewer cells than the parent strain colonies (6.4 vs. 7.8 log CFU/colony). Based on 351 these results, it was hypothesized that the small colony appearance of Lm96_84d_sm on agar 352 plates at $37^{\circ} \mathrm{C}$, was an artefact of having a reduced growth rate at higher temperatures $\left(>25^{\circ} \mathrm{C}\right)$. 353 To test this hypothesis, both variants were grown on $\mathrm{BHI}$ agar plates incubated at $4^{\circ} \mathrm{C}$ and 
354 observed after 3 weeks. As suspected, at $4^{\circ} \mathrm{C}$ no difference in colony size was observed between 355 the two variants.

356 Small colony variants have most thoroughly been studied in Staphylococcus aureus but 357 have also been described in other bacteria including Pseudomonas aeruginosa, Escherichia coli, 358 Vibrio cholera, Salmonella, Lactobacillus acidophilus, and Listeria monocytogenes (Proctor et 359 al., 2006). Small colony variants have reduced growth rates that in some bacteria, have been 360 linked to an inability to produce thymidine caused by mutations in thymidylate synthase or an 361 interruption in the electron transport chain (ETC), specifically resulting from an absence of 362 menadione or hemin biosynthesis and metabolism (Besier et al., 2007; Chatterjee et al., 2008). In

363 L. monocytogenes, studies have found that deletion of a peroxide stress response regulator, perR,

364 results in small colony variants that have increased sensitivity to hydrogen peroxide, and 365 significantly reduced murine virulence (Rea et al., 2005). Curtis et al. (2016) and Christensen et 366 al. (2011), isolated L. monocytogenes small colony variants that had enhanced resistance to 367 several antibiotics and hydrogen peroxide that was a result of mutations in heme biosynthesis 368 and metabolism genes. Furthermore, Van Boeijen et al. (2010) isolated L. monocytogenes small369 colony variants that had enhanced tolerance to heat and high pressure and were found to contain 370 mutations in $c t s R$, encoding a transcriptional repressor of stress response proteins. It is likely that 371 the mutations responsible for enhanced cold tolerance in our variants, also result in decreased 372 metabolism, specifically at warmer temperature $\left(>25^{\circ} \mathrm{C}\right)$.

\subsection{ECT variants exhibited different stress tolerances relative to the parent strain}

The ECT variants were further assessed on their tolerances to other food-related stresses,

375 which may subsequently increase the risk associated with their presence in foods. Stress 376 tolerance profiling was performed on the parent strain and two ECT variants (Table 2): one with 
378 (Lm96_84d_sm) when grown at $37^{\circ} \mathrm{C}$. The first aspect of interest was whether the two variants

379 behaved similarly to the parent strain when cultivated at $25^{\circ} \mathrm{C}$ in $\mathrm{BHIB}$.
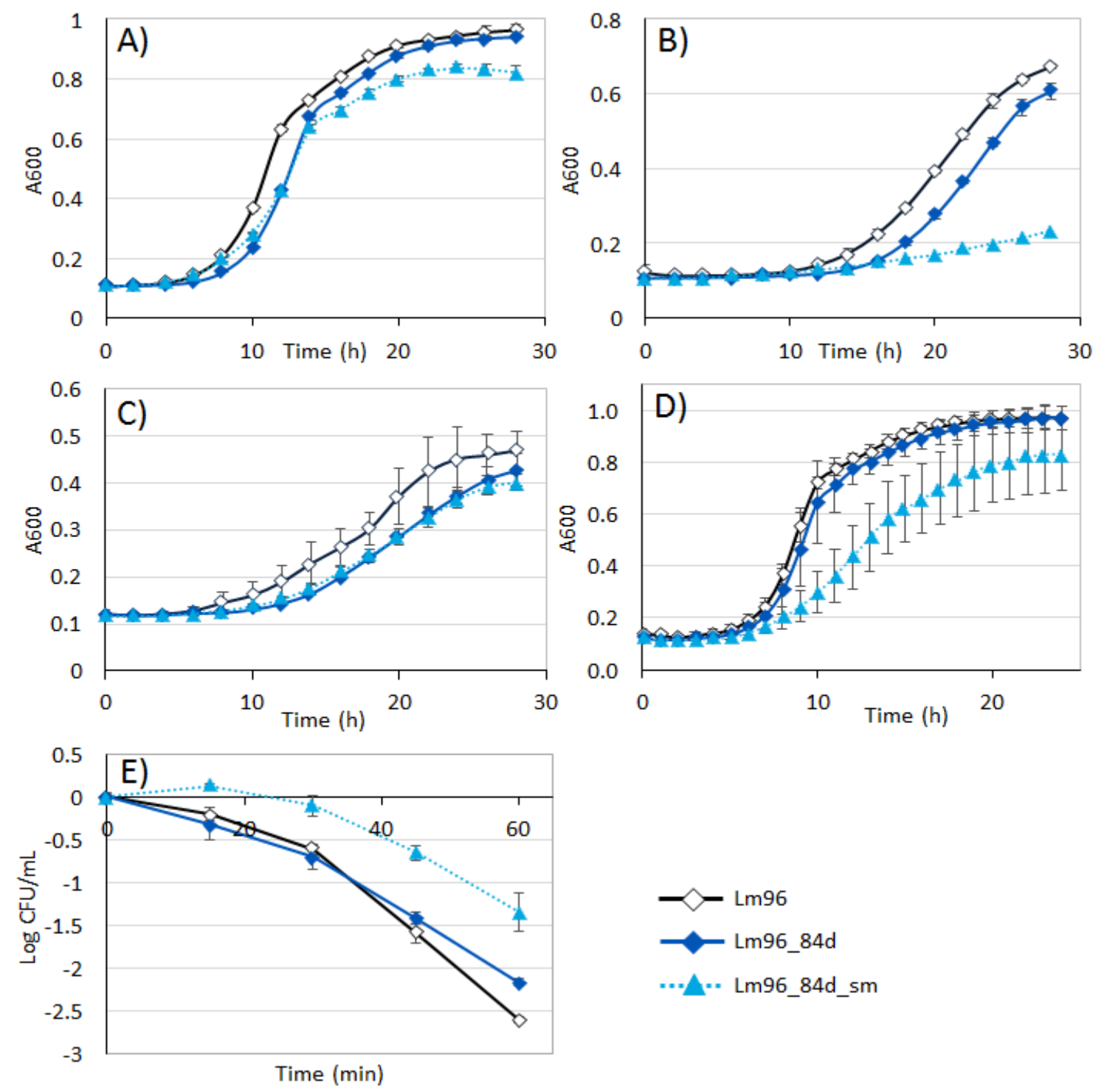

Figure 4. Growth or survival of Lm96 and two enhanced cold-tolerance variants under cold, salt, acid, heat, and desiccation stress. A) Growth in brain heart infusion broth (BHIB) at $25^{\circ} \mathrm{C}, \mathrm{B}$ ) Growth in $\mathrm{BHIB}+6 \% \mathrm{NaCl}$ at $25^{\circ} \mathrm{C}$, C) Growth in BHIB pH 5 at $25^{\circ} \mathrm{C}$, D) re-growth at $25^{\circ} \mathrm{C}$ in BHIB following four days of desiccation $\left(33 \% \mathrm{RH}, 20^{\circ} \mathrm{C}\right.$ ) in buffered peptone water, and $\mathrm{E}$ ) Survival at $52^{\circ} \mathrm{C}$ in peptone saline. Data points denote the averages of replicates and error bars represent standard deviations. All experiments were performed in triplicate with the exception of desiccation which was conducted using six replicates. 
The results (Fig 4A) show that relative to the parent strain, Lm96_84d_sm had a 389 significantly longer lag phase duration (LPD; 7.39 h vs. 6.76 h, p<0.0005), slower maximum 390 growth rate $\left(\mu_{\max } ; 0.071\right.$ vs. $\left.0.086 \mathrm{~A}_{600} / \mathrm{h}, \mathrm{p}<0.005\right)$ and lower maximum cell density $\left(\mathrm{N}_{\max }\right.$; $391 \mathrm{~A}_{600}=0.82$ vs. 0.93, $\left.\mathrm{p}=0.0007\right)$. This was expected given the small colony morphology of this 392 strain when grown at $37^{\circ} \mathrm{C}$ but not when grown at $4^{\circ} \mathrm{C}$. Lm96_84d also had a significantly longer 393 LPD (8-8.36 h, $\mathrm{p} \leq 0.006)$ relative to the parent strain, but had comparable $\mu_{\max }$ and $\mathrm{N}_{\max }$ values 394 (Fig 4A).

Under salt stress conditions Lm96_84d had a significantly longer LPD compared to the 396 parent strain (16.4 vs. $13.9 \mathrm{~h}, \mathrm{p}=0.01$ ) but had a comparable $\mu_{\max }$ and $\mathrm{N}_{\max }$ values (Fig 4B). On 397 the contrary, Lm96_84d_sm had a significantly shorter LPD (10.0 vs. 13.9 h, p=0.002) but a 398 much slower $\mu_{\max }\left(0.007\right.$ vs. $\left.0.046 \mathrm{~A}_{600} / \mathrm{h}, \mathrm{p}<0.0005\right)$ and reduced $\mathrm{N}_{\max }$ after 30 hours $\left(\mathrm{A}_{600}=0.24\right.$ 399 vs. 0.69, $\mathrm{p}<0.0005$ ) compared to the parent strain and Lm96_84d (Fig 4B). While Lm96_84d_sm 400 exhibited a reduced $\mu_{\max }$ and $\mathrm{N}_{\max }$ relative to the parent strain when grown at $25^{\circ} \mathrm{C}$ in regular 401 BHIB, the difference between this variant and the parent strain under salt stress conditions was 402 much greater suggesting that Lm96_84d_sm is in fact more sensitive to salt stress.

403 When grown under acid stress conditions (Fig 4C), Lm96_84d_sm had a significantly 404 longer LPD compared to the parent strain (12.7 h vs. 9.9 h, p=0.044). Lm96_84d_sm also had a 405 longer LPD compared to the parent strain but this difference was not significant. No notable 406 ( $\mathrm{p}>0.05$ ) differences between $\mu_{\max }$ and $\mathrm{N}_{\max }$ values under acid stress conditions were observed. Desiccation survival was primarily evaluated using the time to detectable regrowth 408 (TDR) which corresponds to the lag phase duration of this particular assay. Lm96_84d 409 demonstrated desiccation recovery characteristics comparable to that of the parent strain while 410 Lm96_84d_sm had a significantly longer TDR (7.52 vs. 6.31 h, p<0.0005, Fig 4D) and a slower 
$411 \mu_{\max }\left(0.07\right.$ vs. $\left.0.15 \mathrm{~A}_{600} / \mathrm{h}, \mathrm{p}<0.0005\right)$ relative to the parent strain. This was expected as this

412 variant also had a longer LPD and slower $\mu_{\max }$ when grown at $25^{\circ} \mathrm{C}$ in $\mathrm{BHIB}$. Accordingly, it

413 cannot be concluded that there was an actual difference in the desiccation tolerance of

414 Lm96_84d_sm.

415 When subjected to heat stress at $52^{\circ} \mathrm{C}$, Lm96_84d_sm showed significantly $(\mathrm{p} \leq 0.035)$

416 enhanced survival relative to the parent stain and Lm96_84d at all sampling time points (Fig.

417 4E). Lm96_84d on the other hand, exhibited heat stress survival characteristics comparable to

418 that of the parent strain.

419 Overall, the two ECT variants did not exhibit enhanced survival under salt, acid, or 420 desiccation stress, relative to the parent strain. In fact, at the ambient temperature of $25^{\circ} \mathrm{C}$ they 421 generally demonstrated reduced tolerance to these three stresses with Lm96_84d_sm notably 422 exhibiting severely reduced salt tolerance. These results highlight that mutations beneficial for 423 tolerating one stress can impede tolerance to other stresses. However, this may also occur the 424 other way as demonstrated by Lm96_84d_sm which exhibited both enhanced cold and heat 425 tolerance relative to the parent strain.

426 Whether a mutation will be beneficial for tolerating more than one stress largely depends 427 on the gene(s) affected. For example, a mutation affecting one of the general stress response 428 proteins in L. monocytogenes may subsequently affect the bacterium's ability to tolerate many 429 stresses (Karatzas et al., 2003) while a mutation in a gene used for tolerating a specific stress will 430 have less of an overall impact (Abram et al., 2008). Based on our findings, it appears that 431 Lm96_84d_sm contains a mutation(s) that improves its ability to tolerate both temperature 432 extremes. 


\subsection{ECT variants exhibited different membrane lipid profiles from the parent strain}

Membrane lipid compositional changes are known to be important for bacterial

435 adaptation to changes in temperature. Accordingly, we were interested in determining 1) if the

436 ECT variants have different membrane lipid profiles compared to the parent strain when 437 subjected to cold-stress conditions, and 2) if long-term cold stress survival is associated with a 438 specific membrane-lipid profile.

A) Anteiso C15:0

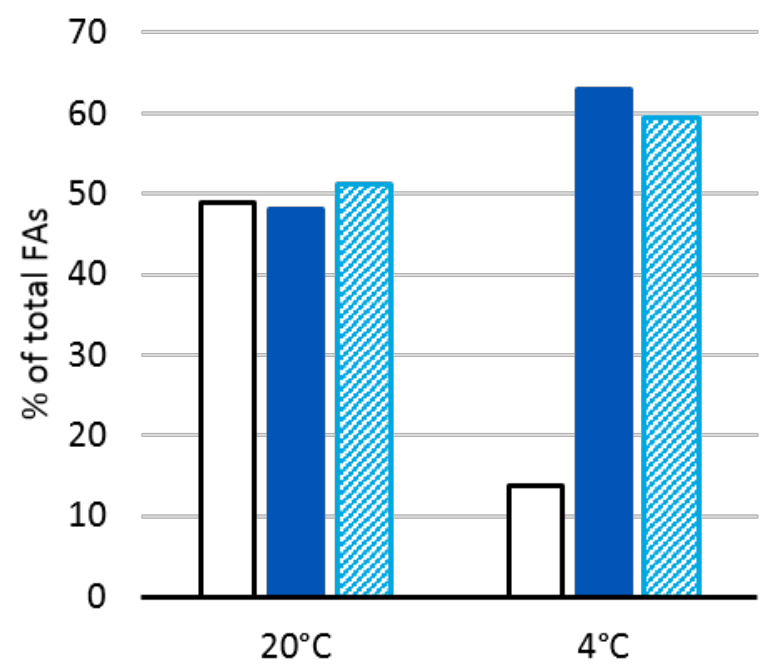

B) $4^{\circ} \mathrm{C}$

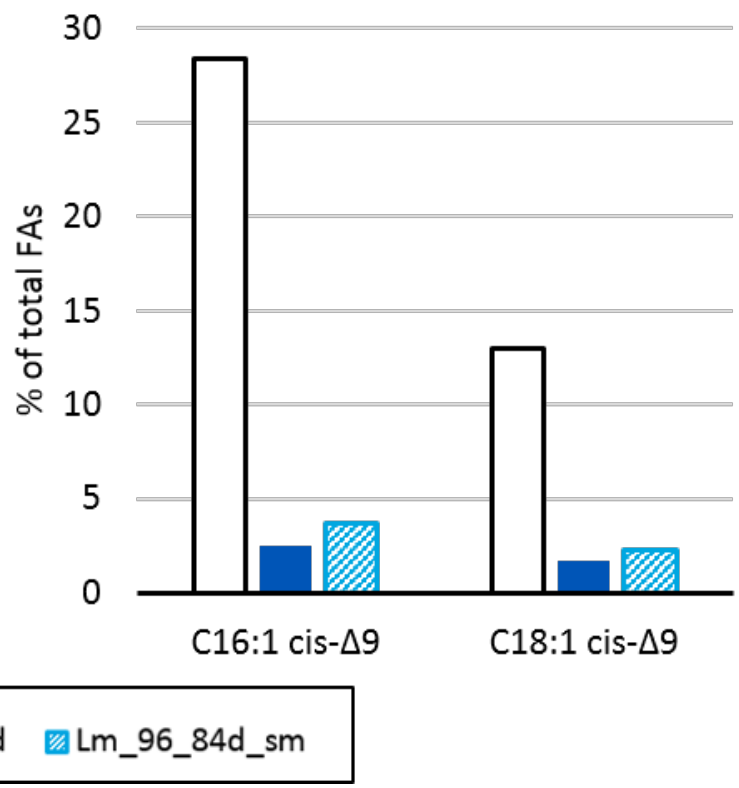

441 Figure 5. Relative proportions of A) anteiso C15:0, and B) C16:1 cis- $\Delta^{9}$ and C18:1 cis- $\Delta^{9}$ in the 442 membranes of exponential-phase cells of Lm96 (parent strain) and two enhanced cold-tolerance 443 variants. Cultures were grown at 20 or $4^{\circ} \mathrm{C}$ in brain heart infusion broth $(n=1)$.

446 variants appeared similar; however, when grown at $4^{\circ} \mathrm{C}$ both ECT variants contained increased 447 levels of anteiso C15:0 (60-62\% vs. 14\%, Fig 5A) and decreased levels of the unsaturated FAs 
448 (UFAs) C16:1 cis- $\Delta^{9}$ (3-4\% vs. 28\% palmitoleic acid) and C18:1 cis- $\Delta^{9}$ (2\% vs. 13\% oleic acid)

449 relative to the parent strain (Fig 5B). Based on previous literature, the cold-sensitive parent strain

450 is the one with the abnormal membrane lipid profile as anteiso C15:0 normally accounts for

451 around $50 \%$ of the membrane lipids in $L$. monocytogenes cells grown at $20-37^{\circ} \mathrm{C}$, and around

$45270 \%$ of the lipids in cells grown at $4^{\circ} \mathrm{C}$ (Annous et al., 1997; Hingston et al., 2017a; Zhu et al.,

453 2005b). Similarly, while palmitoleic and oleic acid are the major unsaturated FAs (UFAs) in the

454 membrane of L. monocytogenes, they are typically present at low levels (0.7-9.15\% collectively).

455 It has been suggested that $L$. monocytogenes can increase its relative proportions of C16:1 cis- $\Delta^{9}$

456 and C18:1 cis- $\Delta^{9}$ to compensate for reduced levels of anteiso C15:0 in its membrane (Hingston et

457 al., 2017a) which is what Lm96 appears to have done. At $4^{\circ} \mathrm{C}$ Lm96 also contained higher levels

458 of other unsaturated and straight-chain fatty acids (SCFAs) under cold stress conditions, 459 including C18:2 cis- $\Delta^{9,13}$, C20:4 cis- $\Delta^{5,8,11,14}$, and C16:0, C17:0, and C18:0 (Table S1). It has

460 been shown that when the membrane of L. monocytogenes contains increased amounts of UFAs

461 (28\% oleic acid), the cell becomes highly susceptible to salt and several antimicrobials (Juneja

462 and Davidson, 1993a). Therefore, it is possible that the reduced growth rate and maximum cell

463 density observed for the parent strain at $4^{\circ} \mathrm{C}$ could be associated with the cells having more

464 permeable membranes.

465 While the two ECT variants had similar membrane lipid profiles, they were not identical.

466 Lm96_84d_sm contained 6-7\% less anteiso C17:0 and 4-5\% less iso C15:0 at 20 C compared to

467 Lm96 and Lm96_84d (Table S1). At higher temperatures (20-37 $\left.{ }^{\circ} \mathrm{C}\right)$ L. monocytogenes is known

468 to contain higher levels of anteiso C17:0 and iso C15:0 compared to when the bacterium is

469 grown at refrigeration temperatures (Juneja and Davidson, 1993b; Kaneda, 1991; Suutari and 470 Laakso, 1992; Zhu et al., 2005a). Therefore, the decreased ability of Lm96_84d_sm to produce 
471 anteiso $\mathrm{C} 17: 0$ and iso $\mathrm{C} 15: 0$ at $20^{\circ} \mathrm{C}$ may be associated with this variant's decreased growth rate

472 and maximum cell density at this temperature. Lm96_84d_sm also contained slightly higher (1-

$4732 \%)$ levels of C12:0, C14:0, C16:0, C18:0, C16:1 $\operatorname{cis} \Delta^{9}$, and C18:1 cis $\Delta^{9}$, and lower levels of iso

474 C16:0 and iso C17:0 at $20^{\circ} \mathrm{C}$. It appears that at $20^{\circ} \mathrm{C}$ Lm96_84d_sm has an increased ability to

475 produce even-numbered SCFAs and monounsaturated FAs, and a decreased ability to make iso 476 FAs.

477 After 354 days of cold storage, the membrane lipid profiles of cells from the long-term 478 cold storage strain cultures, exhibited no outstanding differences from the profiles of cells from 479 the same strains when they were grown to mid-exponential phase ( $\sim 5$ days) at $4^{\circ} \mathrm{C}$ (Table S2). 480 An exception was a $~ 10 \%$ increase in anteiso C15:0 that was observed for Lm1 and Lm20. 481 However, this same increase has been previously observed between Lm1 exponential phase cells 482 grown at $4^{\circ} \mathrm{C}$ and those analyzed 48 hours following the onset of stationary phase at $4^{\circ} \mathrm{C}$ 483 (Hingston et al., 2017a), making it probable that this increase occurred during the onset of 484 stationary phase and is not specifically associated with long-term cold stress survival.

\subsection{ECT variants contained SNPs in acetyl-CoA carboxylase}

Each of the two sequenced ECT variants was found to contain one unique SNP relative to 487 the parent strain. Interestingly, the SNPs were located in adjacent genes $(a c c B, a c c C)$ that encode 488 two of four subunits of the enzyme acetyl-CoA carboxylase (Table 3). The SNP identified in 489 Lm96_84d resulted in a leucine residue being replaced by glutamine in the biotin carboxylase 490 subunit (AccC), and the SNP in Lm96_84d_sm resulted in a serine residue being replaced by 491 phenylalanine in the biotin carboxyl carrier protein (AccB). $a c c B$ and $a c c C$ exist together as a 492 highly conserved operon in many bacteria including Listeria, whereas the genes encoding the 
493 remaining two subunits (AccA, AccD) of acetyl-CoA carboxylase are located elsewhere in the 494 genome (Kondo et al., 1991).

495 Table 3. Single nucleotide polymorphisms identified in L. monocytogenes enhanced cold496 tolerance variants relative to the parent strain (Lm96).

\begin{tabular}{lcc}
\hline & Lm96_84d & Lm96_84d_sm \\
\hline Contig in Lm96 & 10 & 10 \\
Position in Lm96 contig & 360313 & 361625 \\
Contig in variant & 1 & 1 \\
Position in variant contig & 360381 & 361269 \\
Gene affected in Lm96 & BB595_02295 & BB595_02300 \\
Gene affected in variant & RDD56246.1 & RDD58980.1 \\
Position within gene & 1103 & 272 \\
Gene product & Acetyl-CoA carboxylase, biotin & Acetyl-CoA carboxylase, biotin \\
& carboxylase subunit (AccC) & carboxyl carrier protein (AccB) \\
Lm96 codon & cta & tcc \\
Variant codon & caa & ttc \\
Amino acid change & L $\rightarrow \mathrm{Q}$ & S $\rightarrow \mathrm{F}$ \\
\hline
\end{tabular}

Acetyl-CoA carboxylase catalyzes the first step in fatty acid synthesis which is the 499 carboxylation of acetyl-CoA to malonyl-CoA. Accordingly, this enzyme is also considered to be 500 the major rate-limiting enzyme in fatty acid biosynthesis (Cronan and Waldrop, 2002; Zhang and 501 Rock, 2008). Unlike the parent strain, the ECT variants could synthesize adequate amounts of 502 anteiso C15:0. It is therefore logical to assume that these mutations are likely responsible for 503 overcoming this limitation.

504 The SNPs identified in Lm96_84d and Lm96_sm were screened for among 166 L. 505 monocytogenes genomes from our previous study (BioProject PRGNA329415; Hingston et al., 506 2017b). These 166 strains were previously characterized as being tolerant, intermediate, or 507 sensitive to cold, salt, acid, and desiccation stress. All genomes contained a SNP (with respect to 508 Lm96) in the same location in $a c c C$ as Lm96_84d; however, in these strains the SNP resulted in 
509 an arginine residue which differs both from that in the parent strain and in Lm96_84d. The SNP

510 in $a c c B$ in Lm96_84d_sm was unique with regards to the other genomes evaluated, while the

511 allele in the parent strain matched that found in 31 other genomes from our collection. It should

512 be noted that several variations of both $a c c C$ and $a c c B$ existed across the $L$. monocytogenes

513 genomes evaluated, with SNPs occurring in many different locations.

514 Since acetyl-CoA carboxylase plays an essential role in the first step in fatty acid

515 synthesis, one would expect that a malfunction at this step would impede the production of

516 branched-chain FAs as well as SCFAs and UFAs. However, the parent strain was capable of

517 producing both SCFAs and UFAs at $4^{\circ} \mathrm{C}$ and could even produce sufficient amounts of anteiso

$518 \mathrm{C} 15: 0$ at $20^{\circ} \mathrm{C}$, demonstrating that its deficiency to produce branched-chain FAs is cold-stress 519 specific.

520 4. Conclusion

521 In this study, we show that prolonged cold stress exposure can promote the selection of $L$.

522 monocytogenes variants with enhanced cold tolerance (ECT). Twelve ECT variants were

523 successfully isolated from one of 11 strain cultures following 84 days of storage at $4^{\circ} \mathrm{C}$. Two of

524 these variants were selected for further characterization, one of which exhibited a small-colony

525 morphology as a result of a reduced growth rate at warmer temperatures $\left(>25^{\circ} \mathrm{C}\right)$. Interestingly,

526 this variant also demonstrated significantly enhanced heat tolerance at $52^{\circ} \mathrm{C}$ but also reduced salt

527 tolerance at $25^{\circ} \mathrm{C}$ compared to the parent strain and the other variant. Membrane lipid profiling

528 revealed that the cold-sensitive parent strain was unable to produce anteiso fatty acids under

529 cold-stress conditions while the ECT variants were able to overcome this limitation. Both ECT

530 variants contained a single non-synonymous SNP in one of two adjacent genes encoding 
531 subunits of the enzyme acetyl-CoA carboxylase, which is responsible for catalyzing the first step

532 in fatty acid synthesis. Therefore, it is reasonable to propose that these mutations are associated

533 with the improved ability of the ECT variants to produce anteiso FAs, though the mechanisms

534 behind this anomaly are yet to be determined.

While the major findings from this study stemmed from an unusually cold-sensitive strain

536 that is not widely representative of $L$. monocytogenes strains encountered in the food industry,

537 this research has shed light on the types of mutations that can occur in L. monocytogenes that

538 promote enhanced cold tolerance, as well as provided an approximate time period for when such

539 mutations can take place during storage at $4^{\circ} \mathrm{C}$. Furthermore, the study identified genes/enzymes

540 of critical importance to both cold and heat tolerance, and demonstrated the types of membrane-

541 lipid changes that $L$. monocytogenes strains can adopt to survive cold stress.

542 The findings from this study highlight that prolonged refrigeration, which frequently

543 occurs in the food industry, can select for L. monocytogenes variants with enhanced cold

544 tolerance. L. monocytogenes may also acquire such mutations during cold exposure in the natural

545 environment where it is a common inhabitant of soil and waterways. Moreover, we have shown

546 that L. monocytogenes ECT phenotypes can also be associated with enhanced heat tolerance.

547 Notably, this phenotype was also associated with a reduced growth rate at $25^{\circ} \mathrm{C}$, and greatly

548 impaired growth at $25^{\circ} \mathrm{C}$ in $5 \%$ salt. All together this research further emphasizes the role that

549 microbial intervention strategies play in the evolution of bacterial strains and the importance of

550 understanding what these changes may mean for consumer food safety. Future research should

551 continue to investigate the potential impacts of current pathogen control methods (e.g.,

552 preservatives, thermal processing, sanitation) on the evolutionary selection of L. monocytogenes

553 strains with enhanced stress-tolerances. 


\section{Funding}

555 This research was supported by an investment agreement between Alberta Innovates-Bio 556 Solutions and the University of British Columbia [FSC-12-030] as well as the National Sciences 557 and Engineering Research Council of Canada [NSERC Discovery Grant RGPIN-2015-04871]. 558 Author Patricia Hingston was funded by an NSERC CGS D Scholarship.

\section{Acknowledgements}

560 We thank Ms. Lorraine McIntyre at BC Centre for Disease Control and Dr. Taurai Tasara at 561 University of Zurich for providing some of strains used in the long-term cold stress exposure 562 study.

\section{References}

564 Abram, F., Starr, E., Karatzas, K.A.G., Matlawska-Wasowska, K., Boyd, A., Wiedmann, M., 565 Boor, K.J., Connally, D., O’Byrne, C.P., 2008. Identification of components of the sigma B 566 regulon in Listeria monocytogenes that contribute to acid and salt tolerance. Appl. Environ. 567 Microbiol. 74, 6848-58. https://doi.org/10.1128/AEM.00442-08

568 Annous, B.A., Becker, L.A., Bayles, D.O., Labeda, D.P., Wilkinson, B.J., 1997. Critical role of 569 anteiso-C15:0 fatty acid in the growth of Listeria monocytogenes at low temperatures. Appl. $570 \quad$ Environ. Microbiol. 63, 3887-3894.

571 Bankevich, A., Nurk, S., Antipov, D., Gurevich, A.A., Dvorkin, M., Kulikov, A.S., Lesin, V.M., 572 Nikolenko, S.I., Pham, S., Prjibelski, A.D., 2012. SPAdes: a new genome assembly 573 algorithm and its applications to single-cell sequencing. J. Comput. Biol. 19, 455-477.

574 Baranyi, J., Roberts, T.A., 1994. A dynamic approach to predicting bacterial growth in food. Int. 
576 Besier, S., Ludwig, A., Ohlsen, K., Brade, V., Wichelhaus, T. A., 2007. Molecular analysis of 577 the thymidine-auxotrophic small colony variant phenotype of Staphylococcus aureus. Int. J. 578 Med. Microbiol. 297, 217-225. doi: 10.1016/j.ijmm.2007.02.003

579 Boe, L., Danielsen, M., Knudsen, S., Petersen, J.B., Maymann, J., Jensen, P.R., 2000. The 580 frequency of mutators in populations of Escherichia coli. Mutat. Res. Mol. Mech. Mutagen. 448, 47-55. https://doi.org/10.1016/S0027-5107(99)00239-0

Bruno, J.C., Freitag, N.E., 2011. Listeria monocytogenes adapts to long-term stationary phase survival without compromising bacterial virulence. FEMS Microbiol. Lett. 323, 171-179.

Chatterjee, I., Kriegeskorte, A., Fischer, A., Deiwick, S., Theimann, N., Proctor, R.A., Peters, G., Herrmann, M., Kahl, B.C., 2008. In vivo mutations of thymidylate synthase (encoded by thyA) are responsible for thymidine dependency in clinical small-colony variants of Staphylococcus aureus. J. Bacteriol. 190, 834-842. doi: 10.1128/JB.00912-07

Christensen, E. G., Gram, L., Kastbjerg, V. G., 2011. Sublethal triclosan exposure decreases susceptibility to gentamicin and other aminoglycosides in Listeria monocytogenes. Antimicrob. Agents Chemother. 55, 4064-4071. doi: 10.1128/AAC.00460-11

592 Cole, M.B., Jones, M. V., Holyoak, C., 1990. The effect of pH, salt concentration and temperature on the survival and growth of Listeria monocytogenes. J. Appl. Bacteriol. 69,

595 Cooper, A.L., Dean, A.C.R., Hinshelwood, C., 1968. Factors affecting the growth of bacterial $596 \quad$ colonies on agar plates. Proc. R. Soc. London B Biol. Sci. 171.

597 Cronan, J.E., Waldrop, G.L., 2002. Multi-subunit acetyl-CoA carboxylases. Prog. Lipid Res. 41, 
599 Curtis, T. D., Gram, L., Knudsen, G. M., 2016. The small colony variant of Listeria monocytogenes is more tolerant to antibiotics and has altered survival in RAW 264.7 murine macrophages. Front. Microbiol. 7. https://doi.org/10.3389/fmicb.2016.01056

Dalgaard, P., Koutsoumanis, K., 2001. Comparison of maximum specific growth rates and lag times estimated from absorbance and viable count data by different mathematical models. J. Microbiol. Methods 43, 183-196.

Finkel, S.E., 2006. Long-term survival during stationary phase: evolution and the GASP phenotype. Nat. Rev. Microbiol. 4, 113-120. https://doi.org/10.1038/nrmicro1340

Finkel, S.E., Kolter, R., 1999. Evolution of microbial diversity during prolonged starvation.

609

610

611

612

613

$$
\text { Microbiol. Evol. 96, 4023-4027. }
$$

Hingston, P., Chen, J., Allen, K., Hansen, L.T., Wang, S., 2017a. Strand specific RNAsequencing and membrane lipid profiling reveals growth phase-dependent cold stress response mechanisms in Listeria monocytogenes. PLoS One 12. https://doi.org/10.1371/journal.pone.0180123

Hingston, P., Chen, J., Dhillon, B.K., Laing, C., Bertelli, C., Gannon, V., Tasara, T., Allen, K., Brinkman, F.S.L., Hansen, L.T., Wang, S., 2017b. Genotypes associated with Listeria monocytogenes isolates displaying impaired or enhanced tolerances to cold, salt, acid, or desiccation stress. Front. Microbiol. 8. https://doi.org/10.3389/fmicb.2017.00369

Hingston, P.A., Piercey, M.J., Hansen, L.T., 2015. Genes associated with desiccation and osmotic stress in Listeria monocytogenes as revealed by insertional mutagenesis. Appl. Environ. Microbiol. 81. https://doi.org/10.1128/AEM.01134-15

Imhof, M., Schlotterer, C., 2001. Fitness effects of advantageous mutations in evolving 
Escherichia coli populations. Proc. Natl. Acad. Sci. U. S. A. 98, 1113-7. https://doi.org/10.1073/pnas.98.3.1113

623 James, E.S., Cronan, J.E., 2004. Expression of two Escherichia coli acetyl-CoA carboxylase 624 subunits is autoregulated. J. Biol. Chem. 279, 2520-7. https://doi.org/10.1074/jbc.M311584200

626 Juneja, V.K., Davidson, P.M., 1993a. Influence of altered fatty acid composition on resistance of 627 Listeria monocytogenes to antimicrobials. J. Food Prot. 56, 302-305.

628 Juneja, V.K., Davidson, P.M., 1993b. Influence of temperature on the fatty acid profile of 629 Listeria monocytogenes. J. Rapid Methods Autom. Microbiol. 2, 73-81.

Kahl, B.C., 2014. Small colony variants (SCVs) of Staphylococcus aureus - A bacterial survival strategy. Infect. Genet. Evol. 21, 515-522. https://doi.org/10.1016/J.MEEGID.2013.05.016

Kaneda, T., 1991. Iso- and anteiso-fatty acids in bacteria: biosynthesis, function, and taxonomic significance. Microbiol. Rev. 55, 288-302.

Karatzas, K.A.G., Wouters, J.A., Gahan, C.G.M., Hill, C., Abee, T., Bennik, M.H.J., 2003. The CtsR regulator of Listeria monocytogenes contains a variant glycine repeat region that affects piezotolerance, stress resistance, motility and virulence. Mol. Microbiol. 49, 1227-

Koboldt, D.C., Zhang, Q., Larson, D.E., Shen, D., McLellan, M.D., Lin, L., Miller, C.A., Mardis, E.R., Ding, L., Wilson, R.K., 2012. VarScan 2: Somatic mutation and copy number alteration discovery in cancer by exome sequencing. Genome Res. 22, 568-576. https://doi.org/10.1101/gr.129684.111 carboxylase from Escherichia coli: Gene organization and nucleotide sequence of the biotin 
carboxylase subunit. Biochemistry 88, 9730-9733.

645 Langmead, B., Salzberg, S.L., 2012. Fast gapped-read alignment with Bowtie 2. Nat. Methods 9, $646 \quad 357-359$.

647 Leimer, N., Rachmühl, C., Palheiros Marques, M., Bahlmann, A.S., Furrer, A., Eichenseher, F., 648 Seidl, K., Matt, U., Loessner, M.J., Schuepbach, R.A., Zinkernagel, A.S., 2016. Nonstable 649 Staphylococcus aureus small-colony variants are induced by low pH and sensitized to 650 antimicrobial therapy by phagolysosomal alkalinization. J. Infect. Dis. 213, 305-313.

652 Ochiai, Y., Yamada, F., Yoshikawa, Y., Mochizuki, M., Takano, T., Hondo, R., Ueda, F., 2017, 653 Sequential transition of the injury phenotype, temperature-dependent survival and

Proctor, R.A., Von Eiff, C., Kahl, B.C., Becker, K., McNamara, P., Herrmann, M., Peters, G., 2006. Small colony variants: a pathogenic form of bacteria that facilitates persistent and

Schujman, G.E., Mendoza, D. de, 2005. Transcriptional control of membrane lipid synthesis in 664 bacteria. Curr. Opin. Microbiol. 8, 149-153. https://doi.org/10.1016/j.mib.2005.02.003 C.O., Wilkinson, B.J., C, D., 2009. FabH selectivity for anteiso branched-chain fatty acid 
precursors in low-temperature adaptation in Listeria monocytogenes. FEMS Microbiol. Lett. 301, 188-192. https://doi.org/10.1111/j.1574-6968.2009.01814.x

Suutari, M., Laakso, S., 1992. Unsaturated and branched chain-fatty acids in temperature 670 adaptation of Bacillus subtilis and Bacillus megaterium. Biochim. Biophys. Acta (BBA)$671 \quad$ Lipids Lipid Metab. 1126, 119-124.

672 Tatusova, T., DiCuccio, M., Badretdin, A., Chetvernin, V., Nawrocki, E.P., Zaslavsky, L., 673 Lomsadze, A., Pruitt, K.D., Borodovsky, M., Ostell, J., 2016. NCBI prokaryotic genome

US CDC, 2017. Listeria Outbreaks. URL http://www.cdc.gov/listeria/outbreaks/index.html annotation pipeline. Nucleic Acids Res. 44, 6614-6624. https://doi.org/10.1093/nar/gkw569 accessed Jan 15, 2019

Van Boeijen, I.K.H., Chavaroche, A.A.E., Valderrama, W.B., Moezelaar, R., Zwietering, M.H., Abee, T., 2010. Population diversity of Listeria monocytogenes LO28: phenotypic and genotypic characterization of variants resistant to high hydrostatic pressure. Appl. Environ.

Walker, S.J., Archer, P., Banks, J.G., 1990. Growth of Listeria monocytogenes at refrigeration Microbiol. 76, 2225-33. https://doi.org/10.1128/AEM.02434-09

Zhang, Y.-M., Rock, C.O., 2008. Membrane lipid homeostasis in bacteria. Nat. Rev. Microbiol. 6, 222-233. https://doi.org/10.1038/nrmicro1839

Zhu, K., Bayles, D.O., Xiong, A., Jayaswal, R.K., Wilkinson, B.J., 2005a. Precursor and temperature modulation of fatty acid composition and growth of Listeria monocytogenes cold-sensitive mutants with transposon-interrupted branched-chain $\alpha$-keto acid dehydrogenase. Microbiology 151, 615-623.

Zhu, K., Ding, X., Julotok, M., Wilkinson, B.J., 2005b. Exogenous isoleucine and fatty acid 
690 shortening ensure the high content of anteiso-C15: 0 fatty acid required for low-temperature 691 growth of Listeria monocytogenes. Appl. Environ. Microbiol. 71, 8002-8007.

692 Zinser, E.R., Kolter, R., 2004. Escherichia coli evolution during stationary phase. Res.

693 Microbiol. 155, 328-336. https://doi.org/10.1016/j.resmic.2004.01.014

694

695 